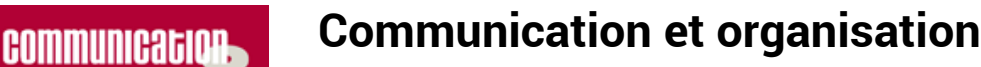

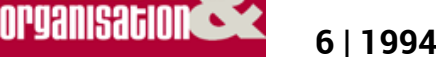

La communication locale

\title{
Les enjeux de la communication en milieu municipal
}

Bernard Dagenais

\section{OpenEdition}

Journals

Édition électronique

URL : http://journals.openedition.org/communicationorganisation/1727

DOI : 10.4000/communicationorganisation. 1727

ISSN : $1775-3546$

Éditeur

Presses universitaires de Bordeaux

Édition imprimée

Date de publication : 1 novembre 1994

ISSN : 1168-5549

Référence électronique

Bernard Dagenais, «Les enjeux de la communication en milieu municipal », Communication et organisation [En ligne], 6 | 1994, mis en ligne le 26 mars 2012, consulté le 21 septembre 2020. URL http://journals.openedition.org/communicationorganisation/1727 ; DOI : https://doi.org/10.4000/ communicationorganisation. 1727

Ce document a été généré automatiquement le 21 septembre 2020.

(c) Presses universitaires de Bordeaux 


\title{
Les enjeux de la communication en milieu municipal
}

\author{
Bernard Dagenais
}

1 Pendant que certains théoriciens de la communication discutent du phénomène de la globalisation de l'information et des autoroutes électroniques, que la publicité a recours, d'un bout à l'autre du monde, aux mêmes concepts, que les enjeux électoraux s'organisent autour de stratégies de mise en marché reprises partout à travers le monde, le citoyen est, d'abord et avant tout, un être solitaire qui vit dans une cellule familiale, qui habite un appartement, une maison, dans une rue, dans un quartier, dans une ville et c'est de là que s'organise la signification concrète de son existence.

2 L'animation des collectivités locales s'articule autour des besoins premiers de l'individu : sa sécurité, sa tranquillité, sa qualité de vie. La municipalité est aussi pour lui un centre de services: les activités culturelles ou sportives, l'enlèvement des ordures ménagères, l'éclairage des rues, l'entretien des routes, l'éducation des enfants. Elle lui offre des activités récréatives, de nature sportive ou culturelle. Et surtout lui propose un cadre de vie.

3 Pour les autorités publiques, la ville est un centre administratif qui doit veiller à la cohésion du groupe par l'imposition de balises et de règles du jeu, par des lois et règlements. Pour maintenir et augmenter la densité de sa population, elle participe activement au développement économique créateur d'emplois. Et celui-ci sera favorisé par une image forte de la ville.

4 La ville est enfin le lieu où s'exprime les ambitions politiques, les querelles idéologiques, les philosophies diverses d'organisation sociale, la diversité des valeurs et les enjeux électoraux locaux.

5 Toutes ces facettes de la réalité municipale s'activent autour de systèmes de communication particuliers à chacune d'elle. Les autorités municipales, par exemple, utilisent diverses techniques de communication pour entretenir le dialogue avec ses administrés. La rencontre personnelle active dans les petites communautés, l'utilisation des journaux municipaux ou la retransmission des séances du conseil municipal sur le câble dans des municipalités plus denses, en passant par les banderoles 
pour annoncer les fêtes populaires et les événements sportifs, constituent différentes approches de communication.

D'autre part, les groupes de citoyens, de commerçants ou d'intérêts divers ont appris à faire valoir leur point de vue sur la place publique en ayant recours à différentes stratégies: pétitions, marches de soutien ou de protestation et feuillets ou lobby intensif.

7 Les médias locaux et régionaux servent enfin de relais aux stratégies des uns et des autres. La vie municipale s'organise parfois par et pour les médias.

Dans cet univers municipal où chaque individu est venu chercher tranquillité, sécurité et un toit pour se protéger des éléments, où chacun revendique une qualité de vie, surgissent aussi les problèmes, de pauvreté, de délinquance et de xénophobie par exemple, qui s'expriment parfois avec une violence insoupçonnée. Là où l'on cherchait la quiétude se développent des conflits de personnalité entre quartiers, entre résidents de souche et nouveaux arrivants, entre gens du pays et immigrants.

9 Comment dès lors, la communication peut-elle servir (ou desservir) la cohésion d'un milieu de vie et la complexité de la réalité municipale? Lorsque l'on considère que celle-ci recouvre l'ensemble des activités d'une collectivité, il faut admettre que la communication est continuellement présente sous des formes extrêmement variées. Car elle traduit à la fois le jeu démocratique des élections, la personnalité et l'image des municipalités, la diversité des services offerts, tout comme la dynamique de toute communauté qui peut osciller entre la quiétude d'une vie de village à la tension délicate d'un milieu en proie à des oppositions diverses.

10 Ce numéro de la revue Communication et organisation sur le thème de la communication en milieu municipal s'ajoute à une longue série de réflexions sur la ville au cours des dernières années. Mentionnons le numéro sur "Communication et sentiment d'appartenance » de la revue Communication et organisation ( $\mathrm{N}^{\circ} 1$, mai 1992), les deux numéros sur "La ville en questions", de la revue Sciences de la société (Cahiers du Lerass, $n^{\circ}$ 30, oct. 1993 et n 31, fév. 1994), le numéro de juin 1994 de la revue Esprit sur le thème de «La jungle des villes». Toutes ces réflexions traduisent une préoccupation bien présente dans l'esprit des décideurs comme des chercheurs : la ville a-t-elle perdu toute dimension humaine et peut-on encore y développer une communauté d'intérêts?

11 Notre intention dans ce numéro est de présenter une série d'études de cas mettant l'accent sur des enjeux particuliers de la communication en milieu municipal et quelques réflexions qui en découlent.

12 À travers l'expérience du tremblement de terre d'octobre 1989 à San Jose, en Californie, et de l'incendie d'un entrepôt de BCP à Saint-Basile-Le-Grand, au Québec, Gilles Willett, de l'Université Laval, nous présente le défi des télécommunications en période de crise. Non seulement elles permettent à l'information structurelle de circuler, mais aussi favorisent la reconstruction symbolique du monde. Dans son article, il illustre le fait que lors d'un sinistre ou d'une catastrophe, c'est tout le tissu structurel et symbolique urbain ainsi que les rituels qui s'effondrent. Dans une telle circonstance, l'action de communiquer et celle de télécommuniquer deviennent les pivots de la restructuration des points de repère et des configurations relationnelles disparus.

13 Pour permettre l'intégration des immigrants dans leur nouveau milieu d'accueil, une expérience particulière a été tentée dans la grande région de la capitale nationale du Canada celle d'utiliser les communications pour sensibiliser les élus municipaux à la 
présence et à l'apport de ces immigrés. C'est cette recherche action que Caroline Andrew et Louise Legault, de l'Université d'Ottawa, présentent en essayant de comprendre de quelle façon et pourquoi les communications ont été au centre de cette intervention.

En voulant valoriser la démocratie municipale, le gouvernement du Québec a adopté un certain nombre de législations au début des années 1980. Alain La vigne, chargé de cours à l'Université du Québec à Rimouski et à l'Université Laval à Québec démontre que les ambitions du législateur ont connu diverses ratés car le discours sur la vie municipale semble ignorer les contraintes des collectivités locales.

C'est dans le même sens que Bernard Dagenais, de l'Université Laval, a voulu démontrer que le monde municipal est un univers hybride où la théorie rejoint parfois difficilement les faits, particulièrement lorsqu'il est question de communication. Faisant état de sa longue expérience de près de 15 ans comme maire d'une petite commune, il explique les grandeurs et les misères de la communication dans un milieu de vie où les rapports de proximité orientent toute l'action politique.

François Demers analyse l'élection municipale de 1989 de la ville de Québec en insistant sur le rôle particulier que peuvent jouer les médias dans l'issue d'une élection que se disputent deux vedettes fortement médiatisées. Il en conclut que les médias, par routine journalistique, adoptent des raccourcis et présentent des points de référence légers, mais qu'en même temps ces pratiques offrent aussi des garanties d'efficacité éprouvée.

17 Pierre Kukuawa, de l'Université de Grenoble, présente une étude qu'il a menée sur l'image des villes de la façade atlantique, expérience qui démontre la nécessité de se créer une image si l'on veut sortir de l'anonymat.

Enfin, Maryse Souchard de l'Université de Cergy-Pontoise et Stéphane Wahnich, directeur de l'Institut d'études et de sondage SCP Communication, en cherchant à définir la spécificité de la communication politique locale, réalisent qu'elle est un outil de pouvoir tout autant qu'un ensemble de techniques visant à animer la vie locale. Mais elle est avant tout un système de représentation visant à (re)créer du collectif dans des milieux de vie de plus en plus différenciés.

19 Ces différentes études illustrent les mythes et la réalité de certaines facettes des communications en milieu municipal. En même temps qu'elles démontrent que la communication est étroitement liée à des enjeux et des défis particuliers qui touchent l'organisation sociale, elles témoignent d'une certaine pauvreté des recherches sur la communication des petites municipalités et des failles que cette situation entraîne dans la construction d'une théorie adéquate de la communication en milieu municipal. 